Proceedings of the 2017 IEEE 24th International Congress on Electronics, Electrical Engineering and Computing, INTERCON 201720 October 2017, Article

number 807966724th IEEE International Congress on Electronics, Electrical Engineering and Computing, INTERCON 2017; Cusco; Peru; 15 August 2017 through 18 August 2017;

Category numberCFP17D62-CDR; Code 131484

\title{
Predictive modeling for presumptive diagnosis of type 2 diabetes mellitus based on symptomatic analysis(Conference Paper)
}

- Barrios, O.Email Author,

- Alberto, D.Email Author,

- Infantes, V.,

- Raphael, E.,

- Aguirre, A.,

- Alexander, J.Email Author

View additional authors

Retrieving additional authors...

- Escuela de Ingeniería de Sistemas y Computación, Universidad Peruana de Ciencias Aplicadas, Peru

View additional affiliations

Retrieving additional affiliations...

Abstract View references (19)

The purpose of using Predictive Modeling for presumptive diagnosis of Type 2 Diabetes Mellitus based on symptomatic analysis is the optimization of the diagnosis phase of the disease through the process of evaluating symptomatic characteristics and daily habits, allowing the forecasting of T2DM without the need of medical exams through predictive analysis. The tool used was SAP Predictive Analytics and in order to identify the most suitable algorithm for the prediction, we evaluated them based on precision and false positive/negative relations, having found the Auto Classification algorithm as the most accurate with a $91.7 \%$ precision and a better correlation between false positives (8) and false negatives (3). (C) 2017 IEEE.

Author keywords

- Auto Classification algorithm 
- diabetes mellitus

- predictive analytics

Indexed keywords

Engineering controlled terms:

DiagnosisOptimizationPredictive analytics

Compendex

Classification algorithmDiabetes mellitusFalse negativesFalse keywords positiveFalse positive/negativePredictive modelingType 2 diabetes mellitus

Engineering main heading:

Computer aided diagnosis

- ISBN: 978-150906362-8

- Source Type: Conference Proceeding

- Original language: English

- DOI: 10.1109/INTERCON.2017.8079667

- Document Type: Conference Paper

- Sponsors: IEEE Peru Section

- Publisher: Institute of Electrical and Electronics Engineers Inc. 